\title{
Design of a WIFI Video Car
}

\author{
Qianqian Zhang ${ }^{1}$, Fengzhi Dai ${ }^{1,2}$ *, Jichao Zhao ${ }^{1}$, Haokang Wen ${ }^{1}$, Hongbo Hao ${ }^{1}$ \\ ${ }^{1}$ Tianjin University of Science and Technology, China; \\ ${ }^{2}$ Tianjin Tianke Intelligent and Manufacture Technology CO., LTD, China \\ E-mail:daifz@tust.edu.cn \\ www.tust.edu.ch
}

\begin{abstract}
This subject is mainly composed of motor, frame, STC89C51 single-chip microcomputer and other auxiliary modules. Through the android client broadcast video car cameras, wireless video signal acquisition by the client send commands to the car at the same time, wireless router transmission instruction by wireless router, single-chip microcomputer is run for processing, then the motors are driven by single chip microcomputer control to rotation and steering, so as to realize the control of motor sports and video acquisition.
\end{abstract}

Keywords: The router OpenWRT, The Socket, WIFI video car, STC89C51, The steering gear

\section{Introduction}

WIFI video car can achieve real-time monitoring, through the computer, mobile phone, tablet etc to connect wireless router, and through the client sends commands to the WIFI router serial port on a router by transmitted to MCU. MCU program parses the command, then operating LN298 driver module so as to achieve the effect of motor control. It has $128 \mathrm{RAM}$ and $4 \mathrm{~K}$ of ROM inside.

\section{Overall scheme design}

In the design and production of circuits, we try to reduce other interference introduced in the measurement to achieve a higher level of performance indicators and functions of electronic products. Usually the circuit design is to take into full account the various factors and the circuit physical.

The circuit mainly involves the connection of router, MCU minimum system, LN298 drive module, steering gear, and camera. Make clear the production task of the system, make specific analysis of the production task, understand the performance of the system, indicators, content and requirements. The important task of the scheme selection is to complete the function of the system according to the knowledge and information, according to the tasks and requirements ${ }^{1}$.

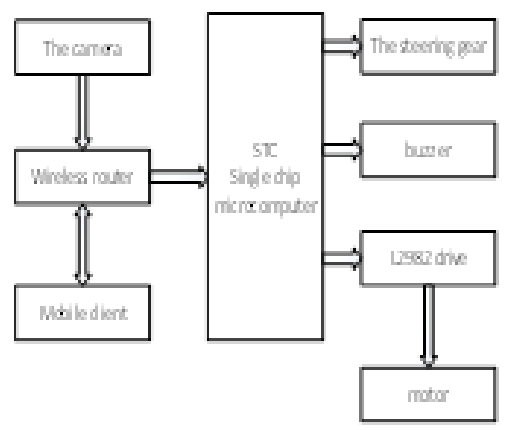

Fig.1. General layout structure

\section{Subsystem design}

\subsection{Circuit design steps}

Economical and detailed circuit design is half the success of our project. First of all, we need to design a minimum system circuit. We need to know which components are

(C) The 2021 International Conference on Artificial Life and Robotics (ICAROB2021), January 21 to 24, 2021 
used in a minimum system circuit. In addition, we need to accurately determine the parameters of various components, such as clock frequency, resistance value, capacitance parameters and so on ${ }^{2}$.

Draw the circuit diagram of the minimum system and simulate it. After the simulation is successful, the circuit welding is carried out. The circuit diagrams are shown in Fig.2 to Fig.4.

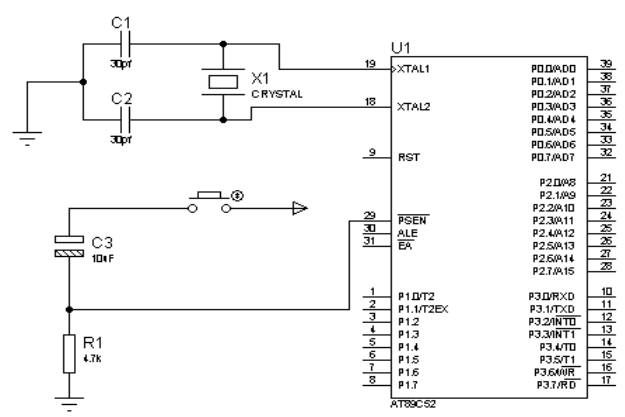

Fig.2. Minimum system simulation circuit

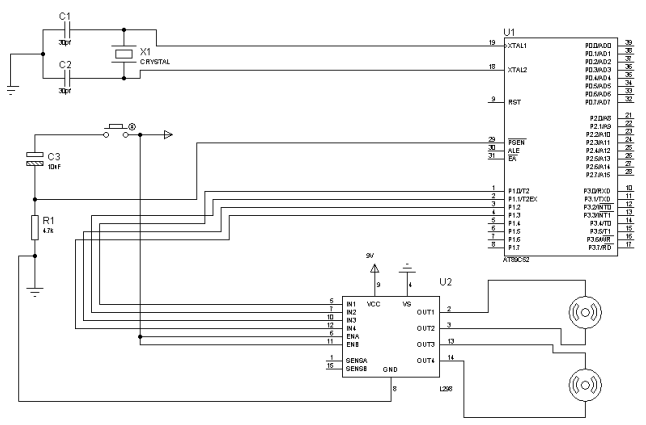

Fig.3. L298N drive module connection circuit

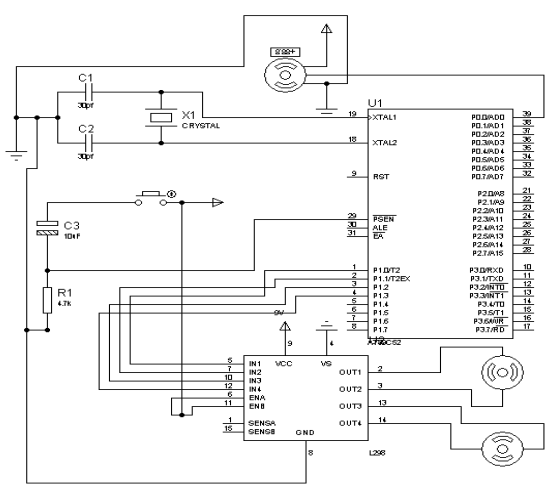

Fig.4. Steering gear connection circuit

Actually, the whole hardware system is not complicated, the main route as the core, openWRT as the main control system. For the next bit machine, 51 of the procedures will be responsible for driving motor and steering gear, we mainly use the two timers, the first is for steering gear, adjust the duty ratio of the steering gear, so that we can through the command code to control the rotation Angle of steering gear ${ }^{3}$.

The second is to use a serial port interrupt, timer is used to set the baud rate (refers to the speed of data transmission) to 9600 , and the timer 2 should set automatic loading mode (refers to give it an initial value). The timer need to be set up only once, after the timer overflow, automatically return to the initial value.

The 298 drive is based on the high and low level connected to the controller pin to determine the positive motor inversion, of course, the drive board is to be in common with the minimum system, which is used to run the program to be effective control driver board, so as to control the motor rotation and steering. When the anode also points to a branch of the steering gear connected to the power supply, in most cases the steering gear is needed, the power converter.

\subsection{Wireless Router}

The wireless router here is actually the same as the one we use in our homes. WIFI is a network protocol, in fact, it is equivalent to a computer, can run the operating system, also need to install the driver. Router connection is shown in Fig.5.

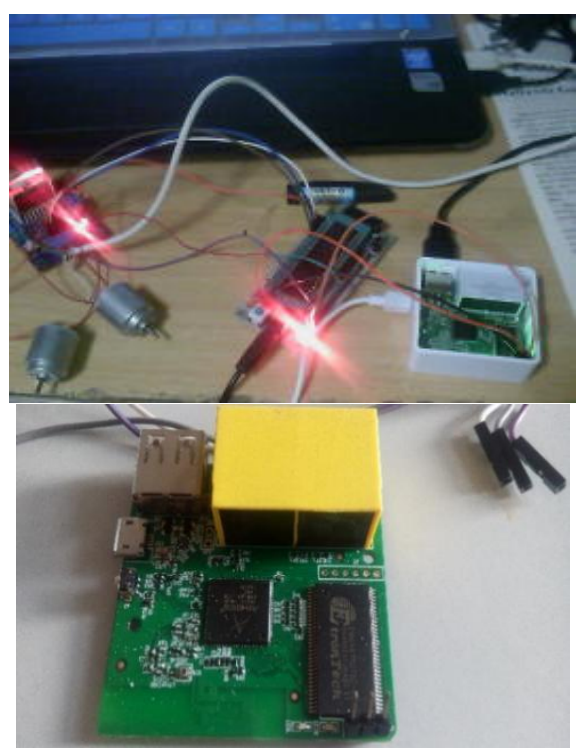

Fig.5. Logic control circuit

\subsection{Principle of steering gear}

The operating principle of steering gear is: in the same period, there are high and low level, high level is the reference signal, generally $1.5 \mathrm{~ms}$, the period is generally $20 \mathrm{~ms}$, which is the duty ratio we often mentioned. The

(C) The 2021 International Conference on Artificial Life and Robotics (ICAROB2021), January 21 to 24, 2021 
difference in voltage between the high and low levels allows the small motor inside to rotate.

Control mode of steering gear is: The control of steering gear generally requires a $20 \mathrm{~ms}$ time-base pulse, and the high level part of the pulse is generally the angle control pulse part within the range of $0.5 \mathrm{~ms} \sim 2.5 \mathrm{~ms}{ }^{4}$. Take the steering gear with an angle of 180 degrees as an example, then the corresponding control relationship is as follows: $0.5 \mathrm{~ms}--0$ degrees; $1.0 \mathrm{~ms}--45$ degrees; $1.5 \mathrm{~ms}$-- 90 degrees; 2.0 ms-- - 135 degrees; 2.5 ms -- 180 degrees; Duty ratio, cycle and signal line of the steering gear are shown in Fig.6.
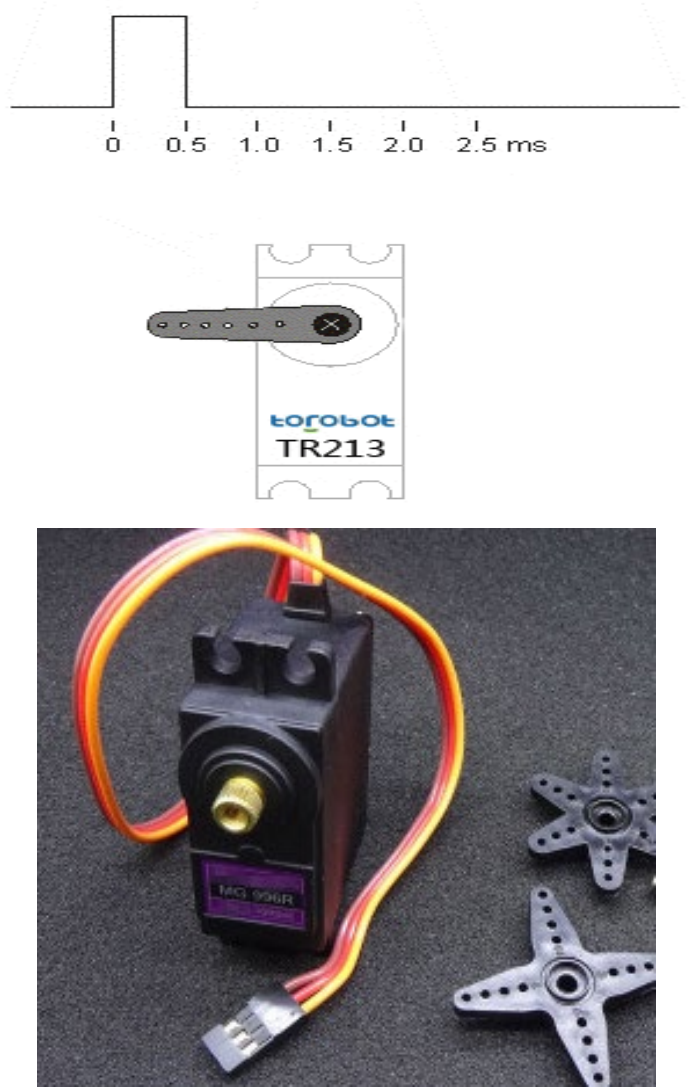

Fig.6. Coplanar camera

\section{The OpenWRT system and construction of development environment}

OpenWRT is a small Linux system, which was originally researched by Apple. However, due to the use of Linux source code, it was forced to open the OpenWRT source code to comply with the GNU Project. That allowed us to use the prototype, which has evolved over time into an operating system commonly used in the embedded space ${ }^{5}$.

Environment setup: OpenWRT is an open source system based on Linux. We can download the source code from its official website. Of course, before developing
OpenWRT, we should first build Linux development environment Ubuntu.

\subsection{Function implementation}

This time, OpenWRT system was used to mainly complete the data acquisition of the camera, and the image was formed after MJPG video streaming software was installed. Through this system to mount the USB interface form of the camera, we use the Onio 12 megapixel camera. This system can receive the command code sent by the mobile phone client, and send it to the next computer through Ser2net software in the way of serial port. So as to achieve the control video car effect.

\subsection{Socket programming}

Socket is just an interface, it's just at the transport level, and of course in order to do network communication, we have to explore the next level, which is the IP level, so there's a lot of connection between network programming and IP addresses, port Numbers.

An IP address is the identity of a host on the Internet. The port number is used to send packets received by the host to that process. Inet aton ( ) is the function used to convert a string referred to by STRPTR into a 32-bit network byte order binary value. The header file is \#include $<$ ARPA /inet.h $>$, inet_addr( ). Same as above, and returns the converted address. The TCP server and client process is shown in Fig.7.

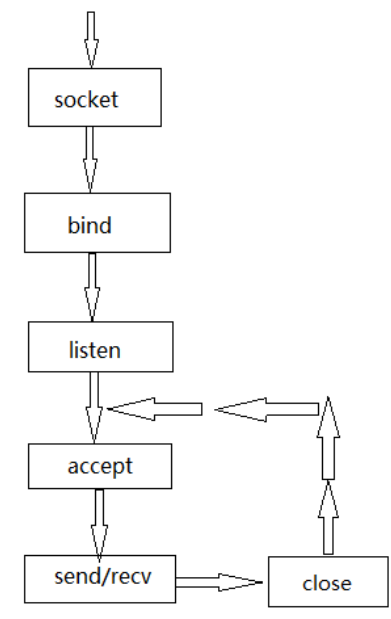

Fig.7. TCP processes

The function socket ( ) creates a socket, bind ( ) binds the address and port, connect( ) establishes a connection, and listen( ) listens for a socket. The function $\operatorname{accept}($ ) accepts connections, $\operatorname{recv}($ ) receives data, and send( ) sends data, close( ) closes the socket. 


\subsection{Description of WIFI video car}

WIFI video car is mainly loaded with router, steering gear, two independent $5 \mathrm{~V}$ power supply, minimal system and camera. The steering gear is used to control the up, down, left and right swing of the camera. This car has been successfully realized ${ }^{5}$.

The mobile client commands are parsed, and the video collection data is sent to the mobile phone, and the mobile real-time monitoring system is successfully completed. WIFI video car is shown in Fig.8.

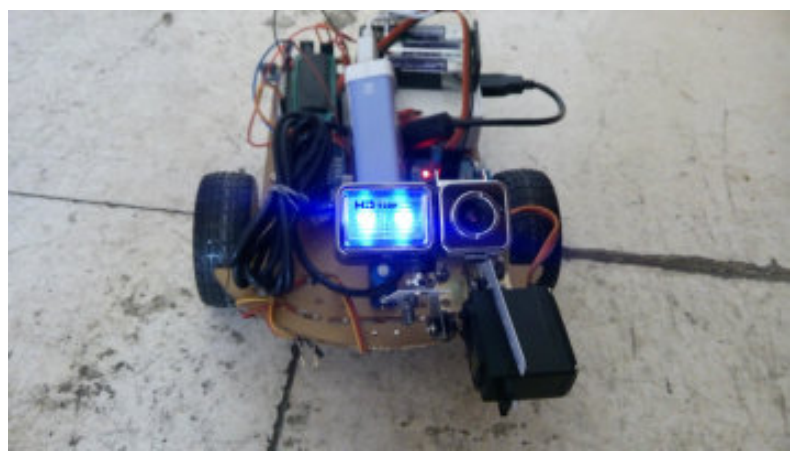

Fig.8. WiFi video car object

\section{Conclusion}

Embedded technology is now regarded as an indispensable high technology in the field of electronics. All the experiments in the design and coding are all belong to the category of embedded. In science and technology developed today, embedded single no longer refers to the single chip microcomputer, it has to grow, or even embedded into many pieces, such as embedded hardware engineer, embedded application engineer, embedded systems engineers, embedded driver engineer.

Our design involves the application layer and hardware, also used in the system, embedded development. Further, we should add a voice transmission function, thus realize the audio/video synchronization.

\section{References}

1. Li Chaoqing. Principle and Interface Technology of Single Chip Microcomputer. Beijing University of Aeronautics and Astronautics Press, 2013.

2. li shengli. Fundamentals of analog electronics technology. School of electrical and electronic engineering, Wuhan institute of engineering, 2013, 13.

3. Yuan Zengmin. Basic Course of Analog Electronics technology.Beijing: Electronics Teaching and Research Group, Tsinghua University, 2014.

4. Song jingbin.Linux network programming (2nd edition). Beijing: Tsinghua University Press, 2014, 2.
5. Liu gang, zhao jianchuan. Linux system transplantation (second edition). Beijing: Tsinghua University press, 2014, 2. 